\title{
Effect of the metal-to-wire ratio on the high-frequency magnetoimpedance of glass-coated CoFeBSi amorphous microwires
}

\author{
R. Valenzuela I, A. Fessant, J. Gieraltowski and C. Tannous \\ Laboratoire de Magnétisme de Bretagne, CNRS-FRE 2697, \\ Université de Bretagne Occidentale, BP 809, Brest CEDEX, 29285, France \\ \Departamento de Materiales Metálicos y Cerámicos, \\ Instituto de Investigaciones en Materiales, Universidad Nacional Autónoma de México, \\ P.O. Box 70-360, Coyoacán, Mexico D.F., 04510, Mexico
}

\begin{abstract}
High frequency $[1-500 \mathrm{MHz}]$ measurements of the magnetoimpedance (MI) of glass-coated $\mathrm{Co}_{69.4} \mathrm{Fe}_{3.7} \mathrm{~B}_{15.9} \mathrm{Si}_{11}$ microwires are carried out with various metal-to-wire diameter ratios. A twinpeak, anhysteretic behaviour is observed as a function of magnetic field. A maximum in the normalized impedance, $\Delta Z / Z$, appears at different values of the frequency $f, 125,140$ and $85 \mathrm{MHz}$ with the corresponding diameter ratio $p=0.80,0.55$ and 0.32 . We describe the measurement technique and interpret our results with a thermodynamic model that leads to a clearer view of the effects of $p$ on the maximum value of MI and the anisotropy field. The behavior of the real and imaginary components of impedance is also investigated; they display a resonance that becomes a function of the DC field $H_{D C}$ for values larger or equal to $H_{K}$ the circumferential anisotropy field for each $p$ value. These results are interpreted in terms of a rotation model of the outer shell magnetization.

PACS numbers: $75.50 . \mathrm{Kj} ; 72.15 . \mathrm{Gd} ; 75.30 . \mathrm{Gw} ; 75.80 .+\mathrm{q}$
\end{abstract}

Index Terms-Amorphous magnetic wires. Giant magnetoimpedance. Magnetic anisotropy. Magnetostriction.

\section{INTRODUCTION}

When a ferromagnetic conductor is traversed by a current of low amplitude and high frequency, its impedance, or rather its Magnetoimpedance (MI) can be altered by applying a DC magnetic field. This phenomenon, first described [1] in the 1930's, has been receiving special attention over the last 15 years [2,3] due to its potential technological applications $[4,5]$ in sensors, devices and instruments. Its fundamental physics is also being deeply examined [6]. MI has been observed in a wide variety of materials, geometries and structures, particularly in amorphous wires having diameters of a few hundred microns. Wires with smaller diameters (a few microns) coated with a glass sheath show an increase of the working frequency, and introduce an additional structural feature that alter the physical parameters $[7,8]$.

Since glass exerts some mechanical stress on the metallic wire, a change in the magnetic response is expected; other works have rather investigated the effect of external stress $[9,10]$, as well as that of various annealing methods $[11,12]$. Consequently, it is of interest to finely tune the physical properties through the control of the thickness and nature of the glass sheath. In this paper, we report on MI measurements of Co-rich amorphous microwires with various ratios of the metal-to-wire diameter, in the [1-500 MHz] frequency range, carried out with a novel [13] broadband technique. This technique allows a complete determination of MI as a function of both frequency and magnetic field.

In contrast with most published works, where MI is measured at a single frequency, or at a discrete set of frequencies, this technique provides a quasi-continuous ensemble of results over the 1-500 MHz frequency range. The effects of the thickness of the glass sheath are clearly illustrated and the variation of the anisotropy field $H_{K}$ is evaluated directly as a function of stress.

Additionally, we carried out an analysis of the real and imaginary components of impedance. The resonance character observed can be attributed to the rotation of the outer shell magnetization as described further below. Possible sensor applications are discussed in the conclusion.

\section{MEASUREMENT TECHNIQUES}

Glass-coated amorphous microwires of nominal composition $\mathrm{Co}_{69.4} \mathrm{Fe}_{3.7} \mathrm{~B}_{15.9} \mathrm{Si}_{11}$ were prepared by fast cooling with the Taylor-Ulitovski technique $\underline{\underline{14}}$. Several metal-to-wire ratio values, $p=\phi_{m} / \phi_{w}$, with $\phi_{m}$ the metallic core diameter and $\phi_{w}$ the total wire diameter, were produced and characterized. For values of metal core diameters of 24, 12 and $7 \mu \mathrm{m}$, with corresponding total diameters of $30,21.8$ and $21.9 \mu \mathrm{m}$, we get the ratio $p=0.80,0.55$ and 0.32 , respectively. In order to make electrical contacts, the glass sheath was etched away over a few $\mathrm{mm}$ on both microwire 
ends, with a solution of hydrofluoric acid. Silver paste contacts were then made in order to proceed with the electrical measurements.

MI measurements were carried out in the [1-500 MHz] range, on pieces of microwires $\sim 12 \mathrm{~mm}$ in length, with an HP 8753C Network Analyzer using a novel broadband measurement technique described in [13]. Helmholtz coils served as source of axial DC magnetic fields ranging from -80 Oe to +80 Oe. Ferromagnetic resonance (FMR) measurements were carried out using $3 \mathrm{~mm}$ long samples, in a JEOL JES-RES 3X spectrometer operating at 9.4 GHz (X-band), at room temperature. Non-resonant low-field absorption (LFA) measurements were taken using a JEOL ES-ZCS2 zero-cross sweep unit, which compensates for any magnetic remanence, allowing precise determination of low magnetic fields around zero.

\section{EXPERIMENTAL RESULTS AND MODELING}

The results obtained are plotted in a continuous $3 \mathrm{D}$ representation of $\Delta Z / Z$, with:

$$
\Delta Z / Z=\frac{\left[Z\left(H_{D C}\right)-Z\left(H_{D C}=80 \mathrm{Oe}\right)\right]}{Z\left(H_{D C}=80 \mathrm{Oe}\right)},
$$

where $Z\left(H_{D C}\right)$ is the total impedance modulus $Z=\sqrt{\left(Z^{\prime 2}+Z^{\prime \prime 2}\right)}$, with $Z$ ' the real part and $Z^{\prime \prime}$ the imaginary part of impedance. $Z\left(H_{D C}\right)$ is a function of the DC field, $H_{D C}$ (in Oersteds), and frequency, $f$. The results for $p=0.8$ are shown in Fig. 1. A symmetrical double peak MI plot is obtained as a function of $H_{D C}$; the peaks are associated with $\pm H_{K}$, the circumferential anisotropy field. We obtain $H_{K} \sim 3.5$ Oe and no hysteresis by cycling the DC field $H_{D C}$.

Regarding frequency $f$, the MI shows a maximum of $\sim 250 \%$ at about $100 \mathrm{MHz}$. Similar plots were obtained with the other $p$ ratios, albeit with significant differences in the values of the anisotropy field and peak frequency values. Instead of making a comparison at a single frequency, as typically done, we choose the frequency at which the maximum in $\Delta Z / Z$ appears and compare results as a function of $H_{D C}$, as shown in Fig. 2. Note that the sample with $p=0.32$ exhibits, as a function of field, several peaks that can be associated with a distribution of the anisotropy axis orientation. This introduces a large uncertainty in the numerical value of the anisotropy field $H_{K}$.

Figure 2 shows clear trends in the results: MI response increases as $p$ increases, while the anisotropy field decreases. $p$ indicates the importance of the metal core with respect to the total diameter of the wire and the stress increases as the thickness of the glass sheath increases. During fabrication, glass-coated microwires are subjected to strong stresses, generally proportional to the thickness of the glass coating that varies inversely proportional to $p$. The origin of such stresses can be understood, since glass possesses a smaller thermal contraction coefficient than metals. In the cooling process, the metallic core tends to contract faster and more substantially than the surrounding glass sheath; however glass hampers such contraction.

Torcunov [14] modeled the thermoelastic and quenching stresses that occur in glass-coated wires and evaluated with a thermodynamic model the stress components in terms of their axial $\sigma_{z z}$, radial $\sigma_{r r}$ and azimuthal $\sigma_{\phi \phi}$ components (in a cylindrical system of coordinates $(r, \phi, z)$ with the $z$ direction along the wire axis). The following expressions (providing the Poisson's coefficients of the glass and metal are equivalent $\nu_{g} \sim \nu_{m} \sim 1 / 3$ ) are obtained and adapted to our case:

$$
\begin{aligned}
\sigma_{r r} & =\frac{\epsilon E_{g}\left(1-p^{2}\right)}{\left(\frac{k}{3}+1\right)\left(1-p^{2}\right)+\frac{4 p^{2}}{3}} \\
\sigma_{\phi \phi} & =\sigma_{r r} \\
\sigma_{z z} & =\sigma_{r r} \frac{(k+1)\left(1-p^{2}\right)+2 p^{2}}{k\left(1-p^{2}\right)+p^{2}}
\end{aligned}
$$

where $E_{g}$ is the glass Young modulus, $k=E_{g} / E_{m}$ and $E_{m}$ is the metallic wire Young modulus.

The term $\epsilon$ is given by the difference of the glass and metal expansion coefficients $\alpha_{g}, \alpha_{m}$ (respectively) times the difference of the minimum glass solidification temperature $T^{*}$ and room temperature $T, \epsilon=\left(\alpha_{m}-\alpha_{g}\right)\left(T^{*}-T\right)$.

We apply this variation to the anisotropy field $H_{K}=2 K_{\sigma} / \mu_{0} M_{s}$ with $K_{\sigma}$ the anisotropy constant of the wire under stress $\sigma$. We consider that the latter induces a change in the anisotropy constant according to $K_{\sigma}=K_{(\sigma=0)}-$ $\frac{3}{2} \lambda_{s}\left(\sigma_{z z}-\sigma_{\phi \phi}\right)$ with the additional assumption of no extra applied stress $\left(\mu_{0}, M_{s}\right.$ and $\lambda_{s}$ are vacuum permeability and saturation magnetization and magnetostriction respectively). Using physical parameters of wires [15] with a 
composition $\left(\mathrm{Co}_{0.94} \mathrm{Fe}_{0.06}\right)_{72.5} \mathrm{~B}_{15} \mathrm{Si}_{12.5}$ similar to ours, we get in Fig. 3, a reasonable agreement with the experimental behaviour, despite a faster tapering off of $H_{K}$ at low values of $p$ where we observe experimentally a large uncertainty in the value of $H_{K}$, due to a broad distribution of anisotropy axis orientation.

Since our measurement technique provides also the real and imaginary components of impedance, we now investigate the frequency behavior of the imaginary component of impedance, $X$, for selected values of the DC field, Fig. 4, for $p=0.80$. For high frequencies $(f>100 \mathrm{MHz}), X$ goes through the axis and take negative values; it changes from an inductive to a capacitive character. This is a common feature of resonance phenomena. We observe that for low DC fields, the plots join in a common point $(f \sim 141 \mathrm{MHz}, X \sim 7 \Omega)$; for fields larger than the anisotropy field $\left(H_{K} \sim 4 \mathrm{Oe}\right)$, the resonance frequency becomes a function of the field. A plot of the imaginary part of impedance as a function of the real component, i.e., a Cole-Cole plot, is a more direct evidence of resonance, when the locus of the points forms a full circle, as shown in Fig. 5, for the $p=0.8$ sample. A comparison of Cole-Cole plots for the other $p$ values appear in Fig. 6, for DC field values corresponding to the anisotropy field of each sample. The $p=0.32$ plot exhibits a deformed circle, probably because the high level of mechanical stresses leads to a complex distribution of the anisotropy axis.

High-frequency MI and FMR have led to some confusion in the past $[16,17]$; however, we feel that the differences between FMR and MI are now well established. Recent results show [18] that as frequency increases (in the $200 \mathrm{MHz}$ - $6 \mathrm{GHz}$ range for Co-rich amorphous ribbons), a divergence in the MI response appears with two maxima in the impedance response, corresponding to $\pm H_{K}$ (the value of which remains virtually constant at all frequencies), and the FMR response, which becomes field dependent, with a Larmor relation that depends on the geometry of the sample. FMR experiments in Co-rich amorphous ribbons at even higher frequencies (X-band at $9.4 \mathrm{GHz}$ ) have exhibited both signals clearly resolved [19], the non-resonant low-field absorption (LFA) similar to MI at fields lower than 50 Oe, and the expected FMR absorption at 1,682 Oe. Therefore our present results show the beginning of this separation. The response associated with FMR shows effectively an increase in resonance frequency as the field increases as observed in the case $p=0.80$.

The relationship between the resonance frequency, $f_{R E S}$, and the resonance field $H_{R E S}$, however, shows a $f_{R E S}^{3} \sim$ $H_{R E S}$ dependence that does not fit the Larmor equation for a cylindrical geometry [20]; an FMR experiment at 9.4 $\mathrm{GHz}$ in these wires leads to a resonance field of 1,132 Oe, about two orders of magnitude smaller than an extrapolation of the relationship exhibited in Fig. 7. In order to understand the behaviour of the resonance frequency as a function of the DC field, we follow the work of Panina et al. [21] describing the wire as containing an axial core magnetization and a circumferential magnetization in an outer shell region transverse to the wire axis. From the rotational permeability of the outer shell magnetization, one may define a resonance frequency assuming negligible Landau-Gilbert damping coefficient:

$$
f_{R E S}=\frac{\gamma}{2 \pi} \sqrt{\left[H_{D C} \sin \left(\theta+\theta_{K}\right)+H_{K} \cos ^{2}(\theta)+4 \pi M_{S}\right]\left[H_{D C} \sin \left(\theta+\theta_{K}\right)+H_{K} \cos (2 \theta)\right]}
$$

where $\gamma$ is the gyromagnetic ratio, $\theta$ is the angle the magnetization makes with the circumferential anisotropy axis $(\mathrm{CAA})$ and $\theta_{K}$ the angle the CAA makes with a direction perpendicular to the wire axis [21]. In principle, $\theta$ is determined from the equilibrium condition, however for simplicity we consider the magnetization along the CAA (that means we take $\theta=0$ ) and therefore the simplified resonance formula becomes:

$$
f_{R E S}=\frac{\gamma}{2 \pi} \sqrt{\left[H_{D C} \sin \left(\theta_{K}\right)+H_{K}+4 \pi M_{S}\right]\left[H_{D C} \sin \left(\theta_{K}\right)+H_{K}\right]}
$$

Replacing the $\sin \left(\theta_{K}\right)$ term by its average value (since the CAA fluctuates randomly with respect to the direction perpendicular to the wire axis) in the above formula, we are able to make a direct comparison with the experimental values we obtain for the resonance frequency versus the DC field as displayed in Fig.7. The agreement we obtain is very good given our simplifying assumptions and the fact we have no free parameter other than the average value of $\sin \left(\theta_{K}\right)$. The low field region is a crossover region from the domain relaxation to the magnetization rotation in the shell whereas in the high field region we ought to observe another transition from the magnetization rotation mode to the FMR mode of the axial magnetization precession (Fig. 8).

\section{CONCLUSION}

In conclusion, the measurement of the MI response of microwires with a novel broadband technique provides a satisfactory view of the interplay between different physical phenomena operating in the glass or metal side. In addition to the increase of anisotropy field as $p$ decreases, a larger distribution of $H_{K}$ is observed for small metal 
cores. Besides, an analysis of the real and imaginary components of impedance has been carried out, leading to the observation of a crossover region from the domain relaxation to the magnetization rotation in the outer shell. A second crossover region to the axial magnetization precession FMR at higher fields is observed (see Fig. 8) making the MI a valuable tool to observe and identify various modal transitions in these glass-covered microwires.

Several applications of the present results are possible. One of them is the ability to select or tune the physical properties such as a better microwire might be produced and suited for a specific application. In particular, the presence of a resonant absorption peak at very low fields, that depends on $H_{D C}$, allows the possibility of engineering devices that can be designed for tunable stopband filtering applications at microwave frequencies [22].

\section{Acknowledgments}

The authors acknowledge Prof. M. Vazquez (Spain) for providing the microwire samples; R.V. thanks DGAPAUNAM, Mexico, for partial support through grant PAPIIT IN119603-3.

1 E.P. Harrison, G.L. Turney, H. Rowe, "An impedance magnetometer" Nature 135, 961 (1935).

2 V.E. Makhotkin, B.P. Shurukhin, V.A. Lopatin, P.Y. Marchukov, Y. K. Levin, "Magnetic field sensors based on amorphous ribbons" Sens. Act. A 25-27, 759-762 (1991).

${ }^{3}$ K. Mandal, S.K. Ghatak, "Large magnetoresistance in an amorphous $\mathrm{Co}_{68.1} \mathrm{Fe}_{4.4} \mathrm{Si}_{12.5} \mathrm{~B}_{15}$ ferromagnetic wire" Phys. Rev. B 47, 14233-14236 (1993).

4 K. Mohri, T. Uchiyama and L. V. Panina, "Recent advances of micro magnetic sensor and sensing applications" Sens. Act. A 59, 1-8 (1997).

5 M. Vazquez, M. Knobel, M.L. Sanchez, R. Valenzuela and A.P. Zhukov, "Giant Magnetoimpedance Effect in Soft Magnetic Wires for Sensor Applications" Sens. Act. A 59, 20-29 (1997).

6 M. Knobel, M. Vazquez, L. Kraus, "Giant Magnetoimpedance" in Handbook of Magnetic Materials, Vol.15, Ed. K.H.J. Buschow, ISBN: 0-444-51459-7 (Elsevier Science, Holland, 2003).

7 H. Chiriac and T.A. Ovari. Prog. Mater. Sci. 40, 233 (1996)

8 M. Vázquez and A. Hernando. J. Phys. D. 29, 939 (1996)

9 M. Carara, K.D. Sossmeier, A.D.C. Viegas, J. Geshev, H. Chiriac and R.L. Sommer. J. Appl. Phys. 98, 033902 (2005)

10 L.V. Panina, S.I. Sandacci and D.P. Makhnovskiy. J. Appl. Phys. 97, 013701 (2005)

11 V. Zhukova, A.F. Cobeño, A. Zhukov, J.M. Blanco, S. Puerta, J. Gonzalez and M. Vazquez. J. Non-Cryst. Solids 287, 31 (2001).

12 L. Kraus, M. Knobel, S.N. Kane and H. Chiriac. J. Appl. Phys. 85, 5435 (1999)

13 A. Fessant, J. Gieraltowski, C. Tannous and R. Valenzuela, "A novel broadband measurement method for the magnetoimpedance of ribbons and thin films" J. Mag. Mag. Mat. 272-276, 1871-1872 (2004).

14 A. V. Torcunov, S.A. Baranov and V. S. Larin, "The internal stresses dependence of the magnetic properties of cast amorphous microwires covered with glass insulation" J. Mag. Mag. Mat. 196-197, 835-836 (1999).

15 K. Mohri, F.B. Humphrey, K. Kawashima, K. Kimura, M. Mizutani, "Large Barkhausen and Matteucci effects in FeCoSiB, FeCrSiB and FeNiSiB amorphous wires" IEEE Trans. Mag. 26, 1789-1791 (1990).

16 J.G.S. Duque, C. Gómez-Polo, A. Yelon, P. Ciureanu, A.E.P. de Araujo, and M. Knobel. J. Magn. Magn. Mater. 271, 390 (2004).

17 A. Yelon, D. Ménard, M. Britel, and P. Ciureanu. Appl. Phys. Lett. 69, 3084 (1996).

18 J.M. Barandiarán, A. García-Arribas, and D. de Cos. J. Appl. Phys. 99, 103904 (2006).

19 H. Montiel, G. Alvarez, I. Betancourt, R. Zamorano, and R. Valenzuela. Appl. Phys. Lett. 86, 072503 (2005).

20 C. Kittel. Phys. Rev. 73, 155 (1948).

${ }^{21}$ L.V. Panina, K. Mohri, T. Uchiyama, M. Noda and K Bushida IEEE Trans. Mag. 31, 1249 (1995).

${ }^{22}$ I. Huynen, G. Goglio, D. Vanhoenacker, and A. Vander Vorst. IEEE Microw. Guid. Wave Lett. 9, 401 (1999).

23 A. Zhukov, J. Magn. Magn. Mater. 242-245, 216 (2002). 


\section{FIGURES}

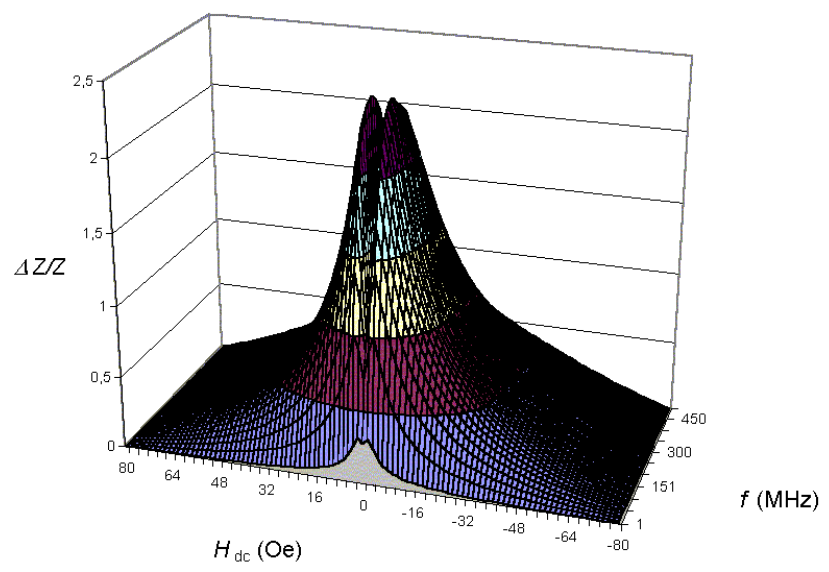

FIG. 1: Magnetoimpedance plot for $p=0.8$ as a function of axial DC field and frequency.

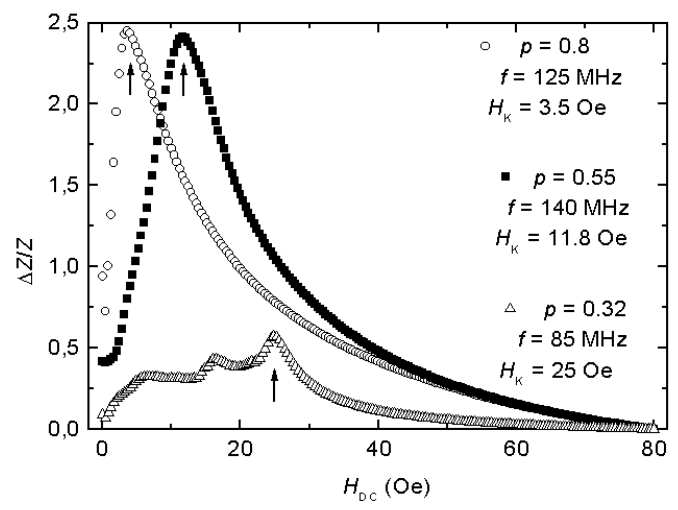

FIG. 2: $\Delta Z / Z$ plot as a function of DC field at the selected frequencies of the MI maximum for each diameter ratio $p$ 


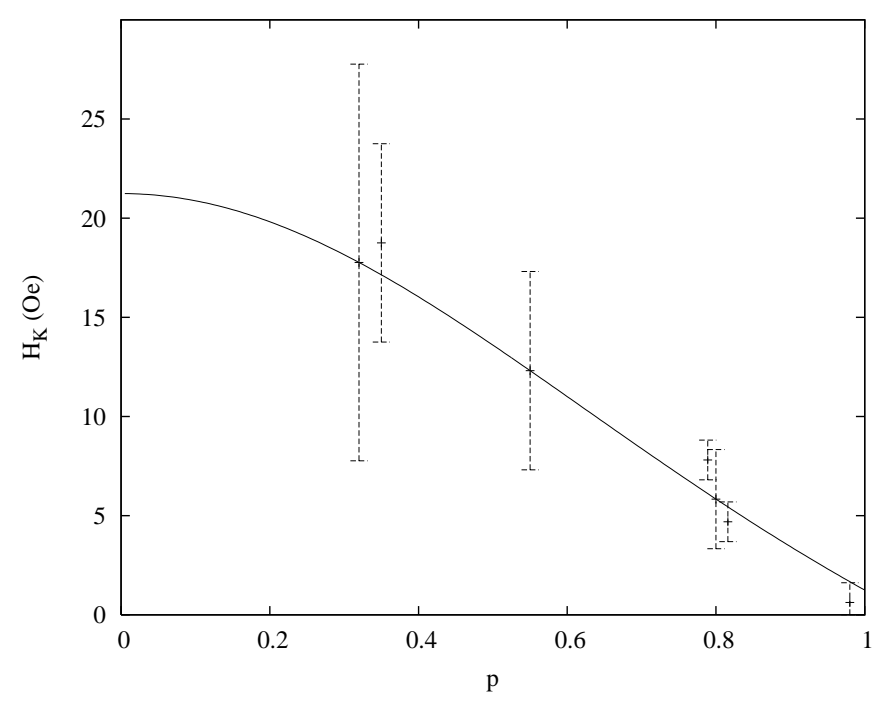

FIG. 3: Effect of the diameter ratio $p$ on the anisotropy field $H_{K}$. Our measured data is for $p=0.32,0.55$ and 0.8 . The $H_{K}$ values corresponding to $p=0.35,0.789,0.816$ and 0.98 are adapted from the literature [23]. The values of $\mu_{0} M_{s}=0.8 \mathrm{~T}$, zero stress anisotropy $K_{(\sigma=0)}=40 \mathrm{~J} / \mathrm{m}^{3}$, and $\lambda_{s}=-0.1 \times 10^{-6}$ are taken from Ref. [15]. Agreement with the theory improves and uncertainty decreases for larger values of $p$.

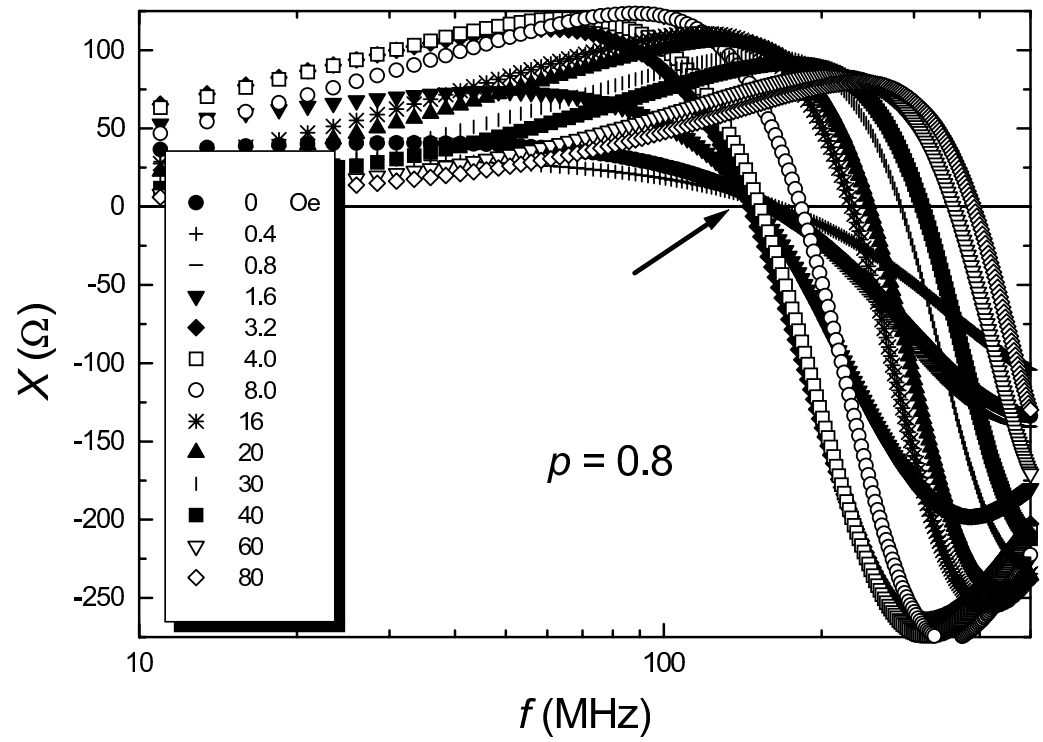

FIG. 4: Imaginary part of impedance as a function of frequency, at selected values of applied field, for the $p=0.8$ sample. The arrow indicates the point where plots merge for low values of field. 


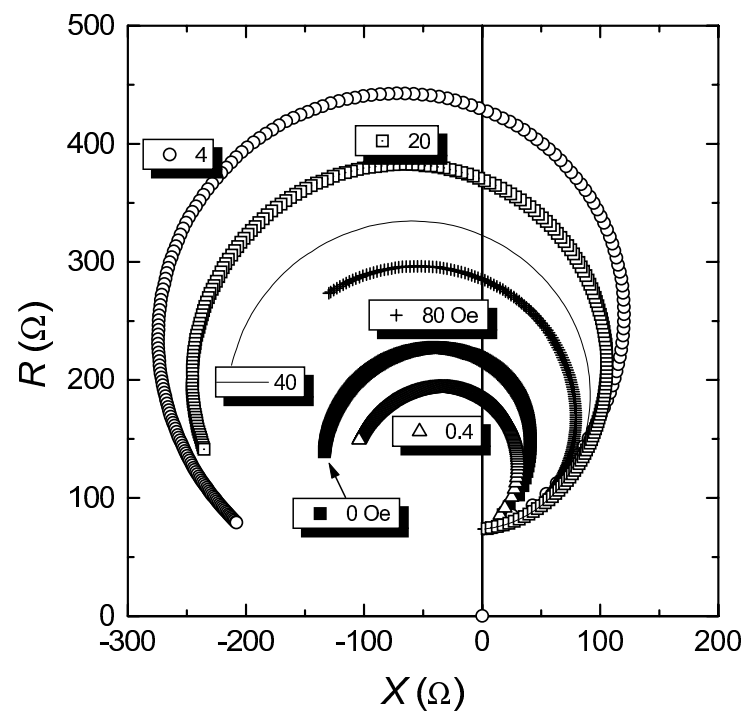

FIG. 5: Cole-Cole plot for the $p=0.8$ sample, for selected values of the applied field.

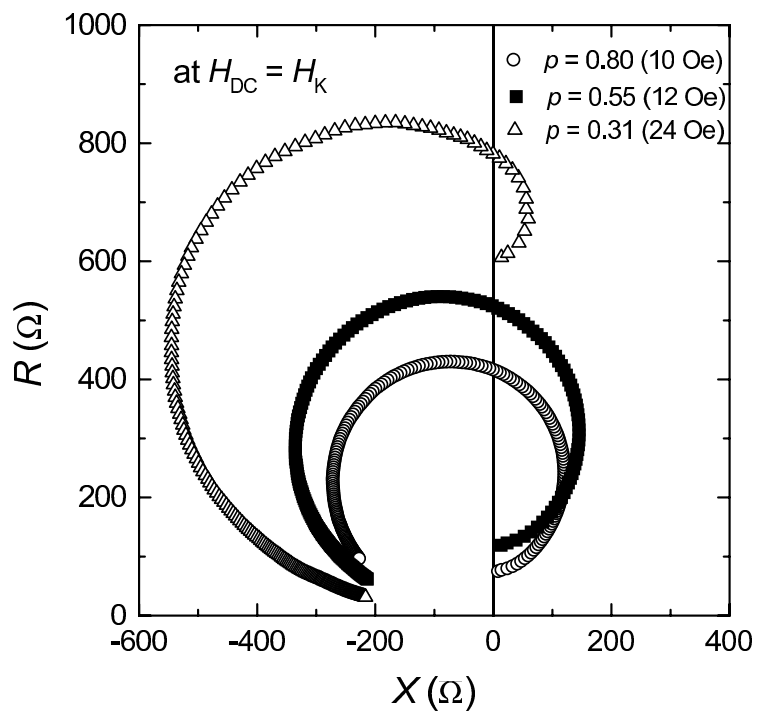

FIG. 6: Cole-Cole plots for the three $p$ values, taken at $H_{D C}$ corresponding to the anisotropy field value $H_{K}$. Note that the $H_{K}$ are slightly different from those indicated in fig.3. $H_{K}$ is taken as 4,12 and 24 Oe for $p=0.8,0.55$ and 0.32 instead of 3.5 , 11.8 and 25 Oe respectively 


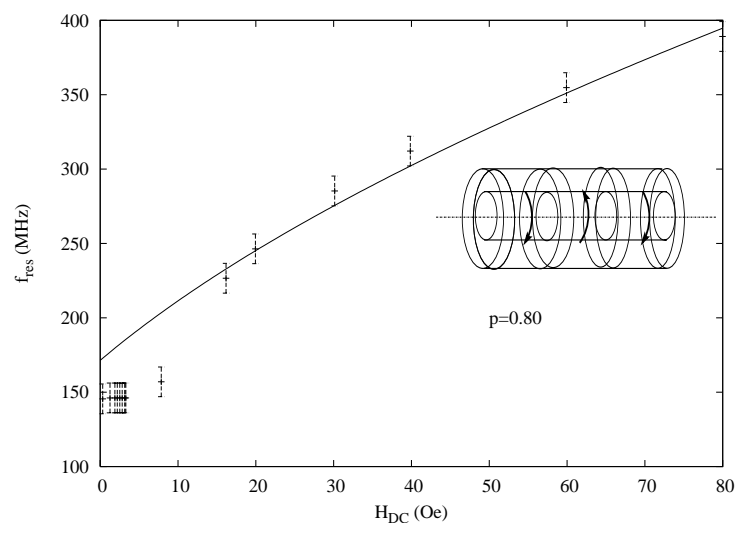

FIG. 7: Comparison between the experimentally determined resonance frequencies (crosses with errorbars) and the theoretical model (continuous line) based on Panina et al's [21] involving rotational motion of the outer shell magnetization as shown in the inset. The low field region [0 Oe $-10 \mathrm{Oe}]$ is a crossover region from domain relaxation to magnetization rotation in the shell.

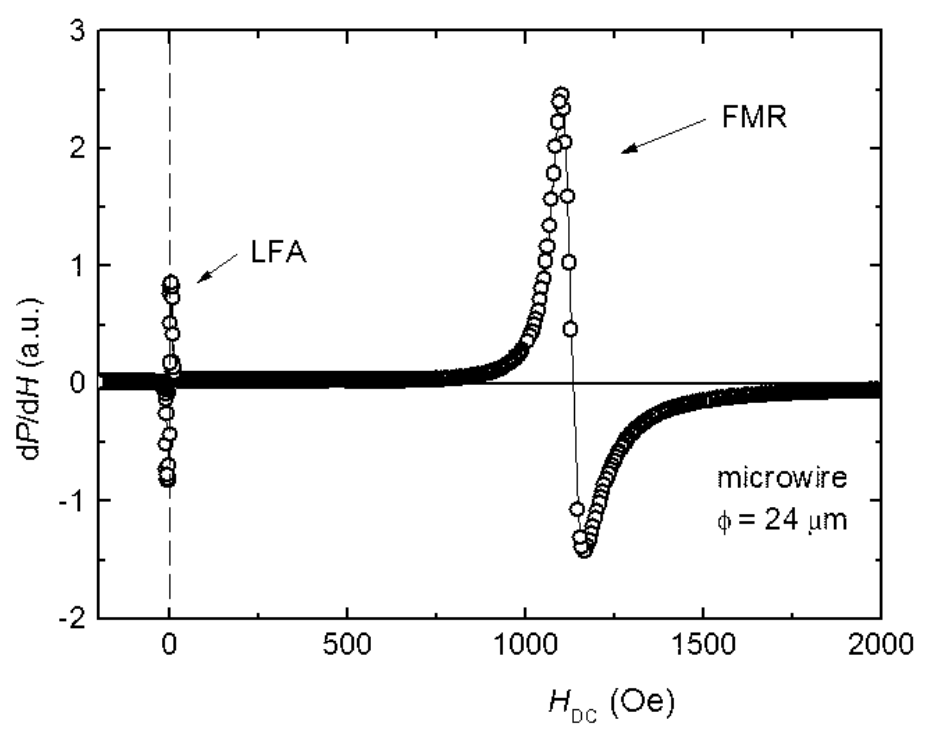

FIG. 8: Microwave absorption of $p=0.8$ microwire at $9.4 \mathrm{GHz}$. The FMR resonance field is 1,132 Oe. A low-field absorption (LFA) double peak appears at $H_{D C} \sim H_{K}=4$ Oe, where MI was observed at low frequencies. The full separation between MI and FMR has therefore taken place. 